\title{
Papers
}

\section{Randomised controlled trial of behavioural infant sleep intervention to improve infant sleep and maternal mood}

H Hiscock, M Wake

\begin{abstract}
Objective To compare the effect of a behavioural sleep intervention with written information about normal sleep on infant sleep problems and maternal depression.

Design Randomised controlled trial.

Setting Well child clinics, Melbourne, Australia

Participants 156 mothers of infants aged 6-12

months with severe sleep problems according to the parents.

Main outcome measures Maternal report of infant sleep problem; scores on Edinburgh postnatal depression scale at two and four months.

Intervention Discussion on behavioural infant sleep intervention (controlled crying) delivered over three consultations.

Results At two months more sleep problems had resolved in the intervention group than in the control group (53/76 v 36/76, $\mathrm{P}=0.005)$. Overall depression scores fell further in the intervention group than in the control group (mean change - 3.7, 95\% confidence interval -4.7 to $-2.7, v-2.5,-1.7$ to $-3.4, \mathrm{P}=0.06$ ). For the subgroup of mothers with depression scores of 10 and over more sleep problems had resolved in the intervention group than in the control group (26/33 $v 13 / 33, \mathrm{P}=0.001)$. In this subgroup depression scores also fell further for intervention mothers than control mothers at two months $(-6.0,-7.5$ to $-4.0, v-3.7,-4.9$ to -2.6 , $\mathrm{P}=0.01)$ and at four months $(-6.5,-7.9$ to $5.1 v-4.2$, -5.9 to $-2.5, \mathrm{P}=0.04)$. By four months, changes in sleep problems and depression scores were similar. Conclusions Behavioural intervention significantly reduces infant sleep problems at two but not four months. Maternal report of symptoms of depression decreased significantly at two months, and this was sustained at four months for mothers with high depression scores.
\end{abstract}

\section{Introduction}

Infant sleep problems and postnatal depression are common. In Australia 36-46\% of parents report a problem with their infant's sleep in the second six months of life, ${ }^{12}$ and $10-15 \%$ of mothers experience postnatal depression in their first year postpartum. ${ }^{3}$ Infant sleep problems and postnatal depression are both associated with increased marital stress, family breakdown, child abuse, child behaviour problems, and maternal anxiety. ${ }^{3}$ Postnatal depression can adversely affect a child's cognitive development. ${ }^{5}$

Postnatal depression is often undetected in primary care. ${ }^{6}$ Even if it is detected, many women are reluctant to accept the diagnosis and to take antidepressant drugs. ${ }^{7}$ Thus there is a large burden of unmanaged postnatal depression in the community. Simple strategies to manage factors linked with depression may decrease this burden. One such factor is infant sleep problems. Commonly used behavioural interventions such as controlled crying and systematic ignoring have been shown to decrease infant sleep problems in randomised controlled trials and to decrease maternal report of symptoms of depression in uncontrolled trials. ${ }^{8}$ However the controlled trials have suffered from small sample sizes (typically 30 to 90 participants), ${ }^{10-12}$ short follow up (under seven weeks), and few or no data about dropout rates. ${ }^{10} 12$ Uncontrolled trials have used non-validated measures of postnatal depression ${ }^{8}$ and small samples derived from clinical settings. ${ }^{8}$

We carried out a randomised controlled trial to determine whether a simple behavioural intervention-controlled crying-would be effective in reducing both sleep problems in infants and symptoms of depression in mothers. We used a reliable validated tool to assess symptoms.

\section{Methods}

Participants

This randomised controlled trial was nested within a larger survey. Between May 1998 and April 1999 all mothers attending routine screening sessions for infant hearing at maternal and child health centres in three local government areas in suburban Melbourne, Australia, were invited to complete a survey about their infant's sleep and their own wellbeing (94\% response rate). About $80 \%$ of children attend these free screening sessions, which are offered to all infants aged 7-9 months. Methods of recruitment and results for this survey have been reported in detail elsewhere. ${ }^{2}$ In summary, $46 \%$ of mothers reported a problem with their infant's sleep. Infants with a sleep problem were more likely to sleep in the parent's bed, be nursed to sleep, take longer to fall asleep, and wake more often and for longer periods overnight. Mothers who were exclusively breast feeding were more likely to report a

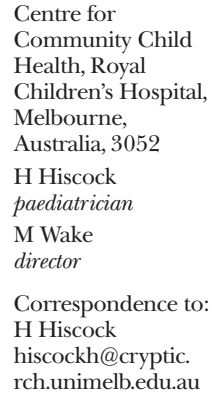

Centre for

Community Child Health, Royal Children's Hospital, Melbourne,

Australia, 3052 H Hiscock paediatrician M Wake director

Correspondence to: H Hiscock hiscockh@cryptic. rch.unimelb.edu.au

bmj.com 2002;324:1062 
sleep problem than those who were not breast feeding or those who gave their infant both formula and breast milk (56\% versus 40\%). Mothers from lower socioeconomic groups were more likely to report sleep problems but other infant and sociodemographic factors did not differ. The presence of an infant sleep problem remained a strong predictor of high depression scores, even after adjustment for known risk factors.

Survey mothers were eligible for the trial if they reported a problem with their infant's sleep and at least one of the following over the preceding two weeks: waking on more than five nights a week,,$^{13}$ waking more than three times a night, ${ }^{13}$ taking more than 30 minutes to fall asleep, ${ }^{14}$ or requiring parental presence to fall asleep. ${ }^{14}$ We excluded mothers with insufficient English to complete questionnaires, who were receiving treatment for postnatal depression, or who reported thoughts of self harm and infants with a major medical or developmental problem and those already receiving help for their sleep problem.

Ethics approval was obtained from the ethics in human research committee of the Royal Children's Hospital, Melbourne, Australia.

\section{Intervention}

Mothers in the intervention group attended three private consultations, held fortnightly at their local maternal and child health centre. $\mathrm{HH}$, a senior paediatric trainee with one year's sleep management experience, conducted the consultations. Sleep management plans were tailored towards individual families. As well as discussing normal sleep cycles, parents were taught that settling after night waking is a learned behaviour that can be modified, infants need to be taught to fall asleep independently, factors reinforcing the sleep problem can be eliminated with appropriate behavioural interventions (see below), an infant's cry may be for more than one reason, and a bedtime routine and consistent daytime naps are desirable.

The main intervention was controlled crying, whereby parents responded to their infant's cry at increasing time intervals, allowing the infant to fall asleep by itself. ${ }^{15}$ A few parents chose "camping out," whereby they sat with their infant until the infant fell asleep and gradually removed their presence over a period of three weeks. Overnight feeding that contributed to night waking was managed by reducing over seven to 10 days the volume of milk given or time taken to feed. When a dummy was causing problems (needing a parent to find and replace it), parents removed it or attached it to the infant's clothing overnight.

Mothers in the intervention group also received a sleep management plan, information about the development and management of sleep problems, and the same information about normal sleep patterns as the control group. They were asked to maintain daily sleep diaries until the first follow up questionnaire.

\section{Control group}

Mothers in the control group were mailed a single sheet describing normal sleep patterns in infants aged 6 to 12 months based on Australian normative data. ${ }^{1}$ This sheet did not include advice on how to manage infant sleep problems.

\section{Process}

Mothers were told that the trial was examining two different ways of managing infant sleep problems to see which one worked best. After the mothers had signed consent forms and completed a baseline questionnaire they were assessed for depression by an independent research assistant, who defined "depressed" as a score over 12 and "not depressed" as a score of 12 and under. ${ }^{16}$ Mothers were then randomised to the intervention or control group within two strata ("depressed" and "not depressed"). Allocation sequences were pregenerated in block sizes of two to 10.

Masking occurred at three points (randomisation, data collection, and analysis). Allocation sequences were concealed from researchers and participants until allocation was complete, and an independent research assistant dealt with all contacts regarding data collection. The principal investigator was blinded to maternal depression scores throughout the trial period.

We measured outcomes at two months and four months after randomisation by mailed questionnaires. The primary outcomes were maternal report of an infant sleep problem (yes or no) and symptoms of depression measured by the Edinburgh postnatal depression scale. ${ }^{16}$ Cut off scores of $>12$ and $\geqslant 10$ have been validated to detect postnatal depression in clinical and community samples respectively. ${ }^{16}{ }^{17}$

Secondary outcomes included severity of infant sleep problem, maternal rating of infant temperament, ${ }^{18}$ marital satisfaction, ${ }^{19}$ maternal stress and coping, ${ }^{20}$ and limits on daily functioning due to maternal emotional or physical health problems. ${ }^{21}$ The last 57 mothers to be recruited completed items about their own quality and quantity of sleep. ${ }^{22}$ We assessed maternal satisfaction with and usefulness of the information on sleep in treating and coping with their infant's sleep problem using $9 \mathrm{~cm}$ visual analogue scales designed for the study. We collected data about helpfulness of the information and strategies provided and extra help sought during the study. Mothers in the intervention group rated the frequency and ease of use of the sleep management strategies.

\section{Analysis}

We calculated that we would need a sample of 140 women to have an $80 \%$ chance of detecting, at a two sided 5\% significance level, a three point difference between the two groups in the mean change in the depression score score, with an assumed SD of $4.8^{23}$ and a loss to follow up of $30 \%$.

We carried out all analyses on an intention to treat basis with Stata version 6 . We compared categorical data with $\chi^{2}$ test and used two sample $t$ tests or MannWhitney $\mathrm{U}$ tests for continuous two group comparisons. Fewer women than anticipated had scores that indicated clinical depression (13 in each group) so we dichotomised depression status at recruitment using community cut off points (depression score $<10$ and $\geqslant 10$ ) for analyses.

We used multiple regression models controlling for group allocation and baseline Edinburgh depression score to assess the impact of seeking additional help on change in depression scores and factors associated 
with increased depression scores at two and four months.

\section{Results}

Participant flow and follow up

Of the 738 mothers who completed the survey, 232 were eligible to participate and left contact details (figure) and 155 of these agreed to participate. Eligible mothers were less likely to participate if their infant was not their first or if the sleep problem was mild. Table 1 shows the baseline variables for the intervention and control groups.

\section{Sleep}

At two months more infant sleep problems had resolved in the intervention group than in the control group (53/76 v 36/76, $\mathrm{P}=0.005$, table 2$)$ and remaining sleep problems were less severe in the intervention group (Mann-Whitney test, $z=-2.46, \mathrm{P}=0.01$ ). In the subgroup of depressed mothers, significantly fewer infants of mothers in the intervention group had a sleep problem at two months (26/33 v 13/33, $\mathrm{P}=0.001$, table 2)

Table 3 shows the sources of extra help sought by mothers for their infant's sleep. At two months more control mothers than intervention mothers had sought extra help (23/76 (30\%) v 9/75 (12\%), $\chi^{2}=7.54$, $\mathrm{P}=0.006)$. Within the control group more mothers who sought extra help reported that their infant's sleep problem had resolved (13/23 (56\%) v 23/53 (43\%), $\left.\chi^{2}=1.11, \mathrm{P}=0.30\right)$.

\section{Maternal depression}

At two months depression scores fell in both groups, with a slightly greater improvement in the intervention group (table 4). After we controlled for additional professional services, Edinburgh depression score, and allocated group with multiple regression the marginally significant fall in depression scores at two

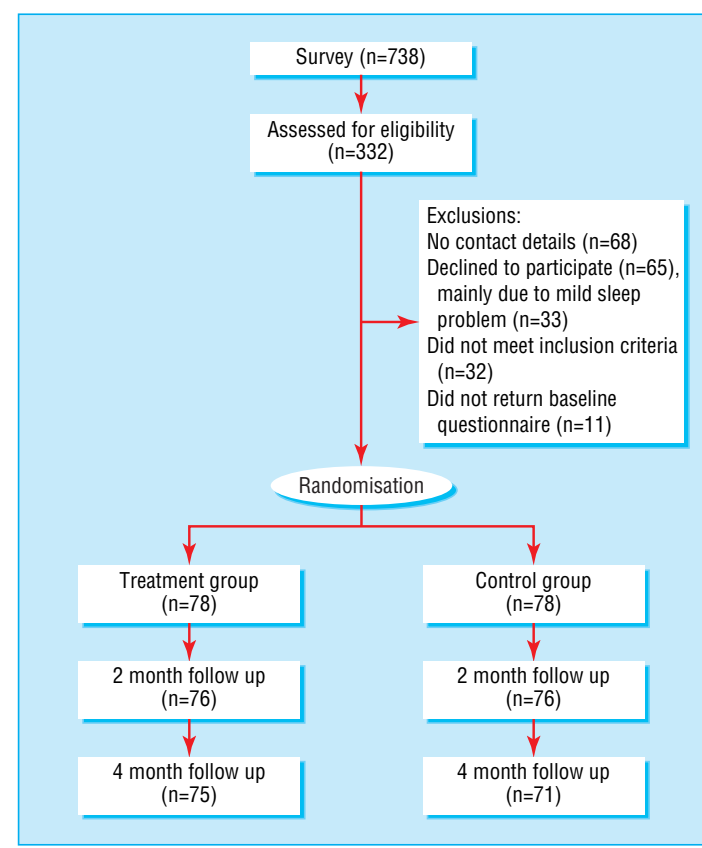

Participant flow chart. All loss to follow up was due to failure to return questionnaires
Table 1 Demographic characteristics of infants and mothers at baseline. Figures are numbers of infants and mothers unless stated otherwise

\begin{tabular}{lcc} 
Variable & $\begin{array}{c}\text { Treatment } \\
(\mathbf{n}=\mathbf{7 8})\end{array}$ & $\begin{array}{c}\text { Control } \\
(\mathbf{n}=\mathbf{7 8})\end{array}$ \\
\hline Infant & & \\
\hline Mean (SD) age (months) & $8.9(0.14)$ & $8.6(0.10)$ \\
\hline Boys & 42 & 47 \\
\hline Girls & 41 & 38 \\
\hline Global temperament score: & 25 & \\
\hline Much easier/easier than average & 37 \\
\hline Average & 14 & 28 \\
\hline More/much more difficult & 2 & 16 \\
\hline Cannot say & & 3 \\
\hline
\end{tabular}

\section{Sleep}

Waking nights per week:

\begin{tabular}{lcc}
\hline $0-3$ & 2 & 9 \\
\hline $4-6$ & 24 & 21 \\
\hline 7 & 52 & 48 \\
\hline Wakings per night: & & \\
\hline $0-1$ & 17 & 21 \\
\hline 2 & 27 & 24 \\
\hline 3 & 21 & 17 \\
\hline$\geqslant 4$ & 13 & 15 \\
\hline Child settled by adult & 45 & 38 \\
\hline Sleep problem severity score: & & \\
\hline $1-2$ (mild) & 9 & 13 \\
\hline $3-4$ (moderate) & 32 & 31 \\
\hline $5-7$ (severe) & 37 & 33 \\
\hline
\end{tabular}

Mother

Edinburgh depression scale score:

\begin{tabular}{lcc}
\hline Mean (SD) & $9.0(0.44)$ & $8.8(0.49)$ \\
\hline$\geqslant 10$ & 44 & 45 \\
\hline Mean (SD) maternal age (years) & $34.1(3.6)$ & $33.3(5.6)$
\end{tabular}

Maternal sleep quantity:

\begin{tabular}{|c|c|c|}
\hline More than enough/enough & 4 & 6 \\
\hline Not quite enough/not nearly enough & 22 & 21 \\
\hline \multicolumn{3}{|l|}{ Maternal sleep quality: } \\
\hline Very good/fairly good & 8 & 8 \\
\hline Fairly bad/very bad & 19 & 22 \\
\hline \multicolumn{3}{|l|}{ Stress rating: } \\
\hline No stress & 2 & 5 \\
\hline Little/some & 71 & 70 \\
\hline Much stress & 5 & 3 \\
\hline \multicolumn{3}{|l|}{ Limits due to physical health problems: } \\
\hline None & 26 & 24 \\
\hline Few/some & 48 & 47 \\
\hline Many & 4 & 7 \\
\hline \multicolumn{3}{|l|}{ Limits due to emotional health problems: } \\
\hline None & 40 & 47 \\
\hline Few/some & 35 & 29 \\
\hline Many & 3 & 2 \\
\hline \multicolumn{3}{|l|}{ Demographic } \\
\hline \multicolumn{3}{|l|}{ Born in Australia: } \\
\hline Mothers & 63 & 62 \\
\hline Fathers & 59 & 59 \\
\hline English spoken at home & 75 & 77 \\
\hline \multicolumn{3}{|l|}{ Marital status: } \\
\hline Single & 1 & 0 \\
\hline Divorced/separated & 1 & 1 \\
\hline Married/cohabiting & 76 & 77 \\
\hline \multicolumn{3}{|l|}{ University education: } \\
\hline Mothers & 55 & 49 \\
\hline Fathers & 50 & 55 \\
\hline \multicolumn{3}{|l|}{ Maternal employment: } \\
\hline Employed & 24 & 22 \\
\hline Home duties/unemployed & 54 & 56 \\
\hline
\end{tabular}

months for the intervention versus control group 
Table 2 Number of mothers whose infants' sleep problems had resolved at two and four months for whole sample and subgroups according to mother's Edinburgh depression score

\begin{tabular}{lcccccc} 
& \multicolumn{2}{c}{ Resolved at two months } & \multicolumn{2}{c}{ Resolved at four months } \\
\hline & Intervention & Control & Pvalue $^{*}$ & Intervention & Control & Pvalue $^{*}$ \\
\hline Whole sample & $53 / 76$ & $36 / 76$ & 0.005 & $48 / 75$ & $39 / 71$ & 0.26 \\
\hline By Edinburgh score: & & & & & & \\
\hline$\geqslant 10$ & $26 / 33$ & $13 / 33$ & 0.001 & $21 / 32$ & $14 / 30$ & 0.13 \\
\hline$<10$ & $27 / 43$ & $22 / 43$ & 0.34 & $27 / 43$ & $25 / 41$ & 0.86 \\
\hline${ }^{*} \chi^{2}$ test. & & & & &
\end{tabular}

Table 3 Numbers of mothers in each group who had sought additional help for their infant's sleep at two months

\begin{tabular}{lcc} 
Source & Control group (n=76) & Intervention group (n=75) \\
\hline Professional & 16 & 5 \\
\hline General practitioner & 6 & 3 \\
\hline Maternal child health nurse & 5 & 1 \\
\hline Mother-baby unit & 5 & 1 \\
\hline Hospital sleep clinic & 0 & 1 \\
\hline Non-professional & 17 & 9 \\
\hline Books & 6 & 2 \\
\hline Friend & 5 & 2 \\
\hline Family member & 3 & 3 \\
\hline Parent group & 3 & 2
\end{tabular}

became clearly significant (point estimate 1.4, 95\% confidence interval 0.2 to $2.5, \mathrm{P}=0.02$ ). By four months the greater fall in depression score for intervention mothers was no longer significant, even when we controlled for extra help. For the subgroup of mothers with initial depression scores $\geqslant 10$, scores fell in both groups with a significantly greater improvement in the intervention group at two and four months (table 4). After we controlled for factors found significantly to alter depression scores in univariate analyses (initial depression score and group membership) the only factor which predicted an increase in depression scores $(\mathrm{P}<0.01$ at two and four months) was a persistent infant sleep problem.

\section{Secondary outcomes}

At two months mothers in the intervention group were more likely than control mothers to rate their own sleep quality as "very good" and less likely to rate it as "very bad" $\left(\chi^{2}=9.93, \mathrm{P}=0.02\right)$, and they were also more likely to have "enough" sleep and less likely to have "not enough" sleep $\left(\chi^{2}=8.11, \mathrm{P}=0.04\right)$. At two months this pattern was repeated in the subgroup of depressed mothers $\left(\chi^{2}=7.58, \mathrm{P}=0.06\right.$ and $\chi^{2}=5.00, \mathrm{P}=0.09$ for group differences in sleep quality and quantity respectively). There were no significant differences in secondary outcomes at four months. At two and four months mothers in the intervention group were as likely to report "no stress" or "little stress" in their lives as were mothers in the control group $(\mathrm{P}=0.37$ and $\mathrm{P}=0.54$ respectively).

Mothers in the intervention group were overwhelmingly more satisfied with the sleep strategies than control mothers were with the written information (median $8.2 v 2.1$ out of a possible 9, $\mathrm{z}=8.82$, $\mathrm{P}<0.001)$. Compared with control mothers, intervention mothers rated the strategies as more useful in both treating their infant's sleep problem (median $8.4 v 1.3$, $\mathrm{z}=9.09, \mathrm{P}<0.001$ ) and coping with it (median $8.2 v 2.0$, $\mathrm{z}=8.65, \mathrm{P}<0.001)$. In the intervention group, $49(65 \%)$ mothers used the strategies "most of the time," 10 (13\%) used them "about half the time," nine (12\%) used them "all the time," and seven (10\%) "a little of the

Table 4 Change in Edinburgh depression scale scores between baseline and two and four months for whole sample and by depression subgroup

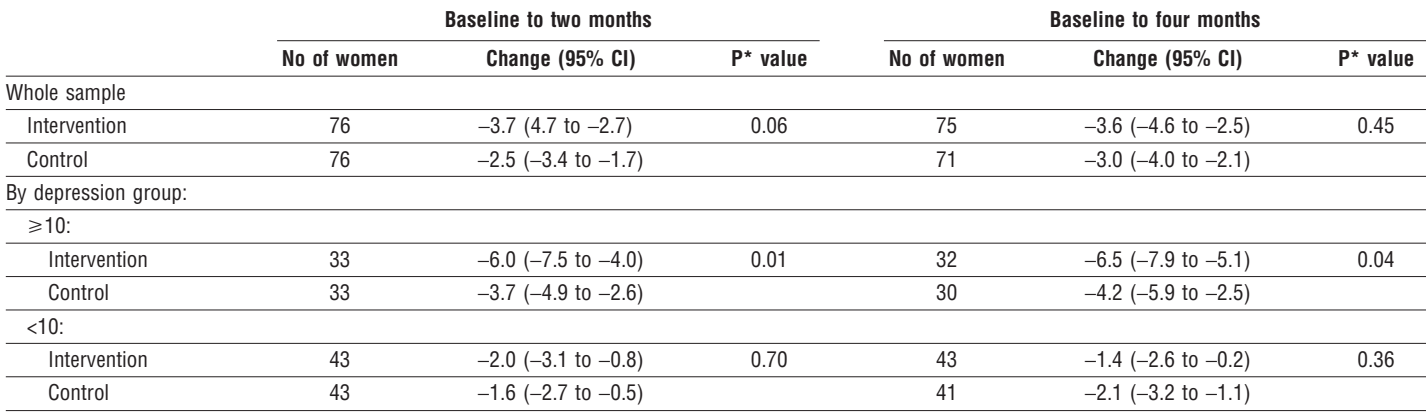

*Student's $t$ test.

Table 5 Helpfulness of strategies and information provided during study. Figures are numbers of mothers

\begin{tabular}{|c|c|c|c|c|c|c|}
\hline \multirow[b]{2}{*}{ Strategy/information } & \multicolumn{3}{|c|}{ Intervention group ( $\mathrm{n}=75$ ) } & \multicolumn{3}{|c|}{ Control group $(n=71)$} \\
\hline & Helpful & Unhelpful & Does not apply & Helpful & Unhelpful & Does not apply \\
\hline Infant sleep patterns ${ }^{*}$ & 73 & 0 & 2 & 36 & 29 & 6 \\
\hline Toddler sleep patterns ${ }^{*}$ & 59 & 6 & 9 & 32 & 27 & 12 \\
\hline Sleep cycles explained & 73 & 0 & 2 & - & - & - \\
\hline Talking to someone & 71 & 2 & 2 & - & - & - \\
\hline Sleep associations explained & 71 & 2 & 2 & - & - & - \\
\hline Controlled crying & 68 & 7 & 0 & - & - & - \\
\hline Using a sleep diary & 67 & 7 & 1 & - & - & - \\
\hline Putting child to bed awake & 57 & 8 & 10 & - & - & - \\
\hline Camping out $\dagger$ & 11 & 7 & 57 & - & - & - \\
\hline
\end{tabular}

*Identical for intervention and control groups

†Used by 17 mothers only. 
time." Table 5 outlines information and strategies mothers found helpful.

\section{Discussion}

A simple behavioural intervention reduced infant sleep problems and maternal symptoms of depression and improved quality and quantity of mothers' sleep in the short term (two months). The same intervention also reduced symptoms of depression at four months for depressed mothers and reduced the amount of help sought from other sources. Use of the intervention did not seem to increase overall stress in a mother's life.

\section{Strengths and weaknesses of the study}

This is the first randomised controlled trial to examine the effect of an infant sleep intervention on both infant sleep and maternal report of depression. Using a validated measure of postnatal depression in a community based sample, we achieved more than $90 \%$ follow up. Although only $67 \%$ of eligible mothers entered the study, those who did not participate were more likely to report only mild sleep problems, suggesting that the intervention did reach nearly all of those really in need. However, our results may not be generalisable to mothers in other socioeconomic groups or those with severe postnatal depression.

Unavoidably, neither the investigator nor the mothers in the study were blind to group membership, which could have led to a bias favouring the intervention. To minimise this, all responses were gathered by written questionnaires and all contacts regarding data collection were with an independent blinded research assistant.

\section{Sleep}

The short term effect of the intervention on infant sleep is similar to that reported in two randomised controlled trials ${ }^{10}{ }^{11}$ and three uncontrolled trials in hospital $\left(84 \% 0^{24}\right.$ to $87 \%{ }^{8}$ sleep problems resolved) and community $\left(83 \%{ }^{14}\right)$ settings. By four months the greater resolution in the intervention group was no longer significant. This is similar to six month findings in a controlled non-randomised study of children aged 4-54 months. ${ }^{25}$ It could have been due to the natural tendency for sleep problems to improve with time $e^{26}$ or to mothers in the intervention group stopping effective behavioural strategies, or both. ${ }^{8}$

\section{Maternal depression}

At two months, depression scores fell by a mean of 6 points $(45 \%)$ for the "depressed" mothers in the intervention group. This is identical with findings of a randomised controlled trial of intensive non-directive counselling sessions delivered by health visitors to 55 women with postnatal depression, which reduced median depression scores by 6 points three months after the intervention. ${ }^{27} \mathrm{~A} 42 \%$ mean reduction in depression scores was reported in an uncontrolled study of 40 women with postnatal depression two months after health visitors taught mothers problem solving techniques for practical problems encountered in infant care in up to eight visits that lasted an hour each. ${ }^{28}$ One uncontrolled sleep intervention study has reported a similar reduction in depression scores. ${ }^{9}$

\section{What is already known on this topic}

Infant sleep problems and postnatal depression are both common potentially serious problems

Women whose infants have sleep problems are more likely to report symptoms of depression

Uncontrolled studies in clinical populations suggest that reducing infant sleep problems improves postnatal depression, but there is no good quality evidence in the community for such effectiveness

\section{What this study adds}

A brief community based sleep intervention based on teaching the controlled crying method effectively decreased infant sleep problems and symptoms of maternal depression, particularly for "depressed" mothers

The intervention was acceptable to mothers and reduced the need for other sources of help

Within the whole sample, depression scores fell by a similar amount in both groups at two and four months. This was partly due to additional help sought by many mothers in the control group. It may also have been due to the known spontaneous improvement in symptoms of depression in mothers over time, ${ }^{27}{ }^{29}$ and as over half $(56 \%)$ of the women in our study had depression scores $<10$ at the start of the trial, a significant fall in their scores was unlikely as they were already low. Finally, repeated completion of the depression scale may have reduced scores.

\section{Conclusions}

This brief community based sleep intervention decreased infant sleep problems and symptoms of maternal depression, particularly for "depressed" mothers. The intervention reduced the need for other professional sleep services, was acceptable to mothers, was of low cost, and was minimally disruptive to families in contrast with many current strategies for postnatal depression. These findings should now be replicated in a larger study in which the intervention is offered and implemented by primary healthcare professionals.

Contributors: The original trial design was formulated by $\mathrm{HH}$ and MW with support from Professor Frank Oberklaid, Director, Centre for Community Child Health, Royal Children's Hospital, Melbourne. The trial was implemented by HH. Data management and analysis was implemented by $\mathrm{HH}$ under guidance from MW and the Clinical Epidemiology and Biostatistics Unit, Royal Children's Hospital, Melbourne. HH produced the first draft of the paper, with redrafting by MW. Both authors will act as guarantors for the paper.

Funding: Research Institute, Royal Children's Hospital, Melbourne, and a Public Health Postgraduate National Health and Medical Research Council Scholarship.

Competing interests: None declared.

1 Armstrong KL, Quinn RA, Dadds MR. The sleep patterns of normal children. Med J Aust 1994;1:202-6.

2 Hiscock H, Wake M. Infant sleep problems and postnatal depression: a community-based study. Pediatrics 2001;107:1317-22.

3 Boyce PM, Stubbs JM. The importance of postnatal depression. Med J Aust 1994;161:471-2. 
4 Kerr SM, Jowett SA. Sleep problems in pre-school children: a review of the literature. Child Care Health Dev 1994;20:379-91.

5 Murray L, Cooper PJ. Effects of postnatal depression on infant development. Arch Dis Child 1997;77:99-101.

6 Hearn G, Iliff A, Jones I, Kirby A, Ormiston P, Parr P, et al. Postnatal depression in the community. Br J Gen Pract 1998;48:1064-6.

7 Appleby L, Warner R, Whitton A, Faragher B. A controlled study of fluoxetine and cognitive-behavioural counselling in the treatment of postnatal depression. BMJ 1997;314:932-6.

8 Leeson R, Barbour J, Romaniuk D, Warr R. Management of infant sleep problems in a residential unit. Child Care Health Dev 1994;20:89-100.

9 Armstrong KL, Van Haeringen AR, Dadds MR, Cash R. Sleep deprivation or postnatal depression in later infancy: separating the chicken from the egg. J Paediatr Child Health 1998;34:260-2.

10 Rickert V, Johnson CM. Reducing nocturnal awakening and crying episodes in infants and young children: a comparison between scheduled awakenings and systematic ignoring. Pediatrics 1988;81:203-12.

11 Seymour F, Brock P, During M, Poole G. Reducing sleep disruptions in young children: evaluation of therapist-guided and written information approaches: a brief report. J Child Psychol Psychiatry 1989;30:913-8.

12 Adams L, Rickert V. Reducing bedtime tantrums: comparison between positive routines and graduated extinction. Pediatrics 1989;84:756-60.

13 Richman N. A community survey of characteristics of one- to two-yearolds with sleep disruptions. J Am Acad Child Psychiatry 1981;20:281-91.

14 Minde K, Popiel K, Leos N, Falkner S, Parker K, Handley-Derry M. The evaluation and treatment of sleep disturbances in young children. J Child Psychol Psychiatry 1993;34:521-33.

15 France KG, Henderson JMT, Hudson S. Fact, act and tact. A three-stage approach to treating the sleep problems of infants and young children. Child Adolesc Clin North Am 1996;5:581-99.

16 Cox JL, Holden JM, Sagovsky R. Detection of postnatal depression. Development of a 10-item Edinburgh postnatal depression scale. $\mathrm{Br} \mathrm{J}$ Psychiatry 1987;150:782-6.

17 Murray L, Carothers AD. The validation of the Edinburgh postnatal depression scale on a community sample. Br J Psychiatry 1990;157:28890.

18 Sanson A, Prior M, Garino E, Oberklaid F, Sewell J. The structure of infant temperament: factor analysis of the revised infant temperament questionnaire. Infant Behav Dev 1987;10:97-104.
19 Sharpley CF, Rogers JH. Preliminary validation of the abbreviated Spanier dyadic adjustment scale: some psychometric data regarding a screening test of marital adjustment. Educ Psychol Measurement 1984;44:1045-9

20 Smith J, Prior M. Temperament and stress resilience in school-age children: a within-families study. J Am Acad Child Adolesc Psychiatry 1995;34:168-79.

21 Ware JE Jr, Nelson EC, Sherbourne CD, Stewart AL. Preliminary tests of a 6-item general health survey: a patient application. In: Stewart AL, Ware JE, eds. Measuring functioning and well-being: the medical outcomes study approach. Durham, NC: Duke University Press, 1992.

22 Buysse D, Reynolds C, Monk T, Berman S, Kupfer D. The Pittsburgh sleep quality index: a new instrument for psychiatric practice and research. Psychiatry Res 1989;28:193-213.

23 Astbury J, Brown S, Lumley J, Small R. Birth events, birth experiences and social differences in postnatal depression. Aust J Public Health $1994 ; 18: 176-84$.

24 Jones DPH, Verduyn CM. Behavioural management of sleep problems. Arch Dis Child 1983;58:442-4.

25 Weir I, Dinnick S. Behaviour modification in the treatment of sleep problems occurring in young children: a controlled trial using health visitors as therapists. Child Care Health Dev 1988;14:355-67.

26 Zuckerman B, Stevenson J, Bailey V. Sleep problems in early childhood: continuities, predictive factors, and behavioral correlates. Pediatrics 1987;80:664-71.

27 Holden JM, Sagovsky R, Cox JL. Counselling in a general practice setting: controlled study of health visitor intervention in the treatment of postnatal depression. BMJ 1989:298:223-6.

28 Seely S, Murray L, Cooper PJ. The outcome for mothers and babies of health visitor intervention. Health Visit 1996;69:135-8.

29 Cooper PJ, Campbell EA, Day A, Kennerley H, Bond A. Non-psychotic psychiatric disorder after childbirth. A prospective study of prevalence, incidence, course, and nature. Br J Psychiatry 1988;152:799-806.

(Accepted 3 December 2001) 